\title{
Research on Reliability Optimization Calculation of Computer Network Based on Genetic Algorithm
}

\author{
Yana Zhang ${ }^{a}$, Xin Cao ${ }^{b}$ \\ Department of Software Engineering, Shijiazhuang Information Engineering Vocational \\ College,Shijiazhuang, 050035, China

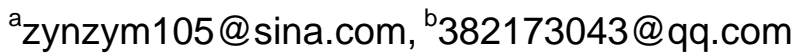

Keywords: .Genetic algorithm; Reliability; Computer network; Optimization; Simulation

Abstract. To decrease network link cost is one of the most important paimsrs for computer network reliability optimization design. In order to make the network cost minimum, the network link medium cost, mathematics mode of reliability and resolve algorithm must be considered when it is designed. In the paper, we present a genetic algorithm used in optimization calculation for minimizing total link-cost and increasing the reliability of the network. Executive procedures of the algorithm are described. The simulation results show that genetic algorithm provides a method to resolves the network reliability optimization problem which normal approach can not resolve. By using the maneuverable algorithm, optimization process is accelerated, efficiency is increased.

\section{Introduction}

The rapid development of computer network technology and its application in various fields spread makes the network user node increasing, the size of the network growing, the network becoming more complex[1]. At the same time, people's dependence on computer networks is growing. Once the computer network system is failure, the loss caused will be huge, even immeasurable. Therefore, network reliability has become an important indicator of the overall performance of the computer network system[2]. How to improve the network reliability, based on reducing the total cost of the network link became a focus of network that designers and users are generally concerned[3]. The traditional method for solving NP optimization problem appeared to be inadequate. In response to this phenomenon, this paper proposed genetic algorithm to conduct reliability optimization of computer network, and used examples to achieve the execution process of the algorithm.

\section{The mathematical model of the problem}

The cost matrix of network link media is defined as Eq. (1), where $C_{0}$ represents the cost matrix;

$c_{i j}$ represents the cost of the link between the node $i$ and node $j$.

$$
C_{0}=\left[\begin{array}{cccc}
c_{11} & c_{12} & \cdots & c_{1 n} \\
c_{21} & c_{22} & \cdots & c_{2 n} \\
\vdots & \vdots & \ddots & \vdots \\
c_{m 1} & c_{m 2} & \cdots & c_{m n}
\end{array}\right]
$$

The mathematical model of network link media costs is shown as follows: 


$$
\begin{aligned}
& \min C=\sum_{i=1}^{N} \sum_{j=1}^{i} c_{i j} g_{i j} \\
& \text { s.t. }\left\{\begin{array}{l}
D_{i} a_{i j} \leq \alpha \quad(i, j=1,2, \cdots, N) \\
\sum_{j=1, j \neq i} g_{i j} \geq \beta \quad(i=1,2, \cdots, N)
\end{array}\right.
\end{aligned}
$$

Where $g_{i j}=\left\{\begin{array}{l}1 \text {, There is a direct link between } i \text { and } j \\ 0 \text {, There is no direct link between } i \text { and } j\end{array}, C\right.$ represents the total cost of the network, $N$ represents the total number of nodes in the network, $D_{i} a_{i j}$ represents the logic shortest link number between node $i$ and node $j$; $\alpha$ and $\beta$ represent the reliability constraints constant of network nodes.

The reliability matrix of network link medium is shown in Eq.(4), where $R_{0}$ represents the reliability matrix; $r_{i j}$ represents the reliability of link medium between node $i$ and node $j$.

$$
R_{0}=\left[\begin{array}{cccc}
r_{11} & r_{12} & \cdots & r_{1 n} \\
r_{21} & r_{22} & \cdots & r_{2 n} \\
\vdots & \vdots & \ddots & \vdots \\
r_{m 1} & r_{m 2} & \cdots & r_{m n}
\end{array}\right]
$$

In $G=(N, L)$, there may be a subset of all nodes which is in a normal state, $L^{\prime} \subseteq L$. The reliability that all the nodes are in normal state is defined as $\prod_{i=1}^{N} P\left(n_{i}\right)$, where $n_{i}$ represents the $i$-th node, $P\left(l_{i}\right)$ represents the reliability of $i$-th link medium.

Computer network reliability optimization problem can be described as abstract: $R$ maximized, while $C \leq C_{0}$. Reliability mathematical model of the entire computer network is established as follows:

$$
R=\sum_{\Omega} \prod_{i \in L^{\prime}} P\left(l_{i}\right) \circ\left\|\prod_{i \in\left(L / L^{\prime}\right)^{\prime}}\left(1-P\left(l_{i}\right)\right)\right\| \circ \prod_{i=1}^{n} P\left(n_{i}\right)
$$

Where $\Omega$ represents all the normal state sets of the network; $l_{i}$ represents the medium of $i$-th link.

\section{Genetic algorithm}

Genetic algorithm (GA)is a kind of random search algorithm based on biological natural selection and genetic stochastic. The optimal point is searched through the group search strategy and the exchange of information between individuals. It has great potential in solving complex optimization 
problems.

\section{The part of GA.}

(1) Gene expression;

(2) Determination of initial population;

(3) Calculate the value of genetic fitness;

(4) Genetic operations (crossover and mutation);

(5) Evolutionary computation (selection).

\section{The steps of GA.}

The solving process of genetic algorithms is shown as follows:

begin

$t \leftarrow 0$;

Initialize $p(t)$;

Evaluate $p(t)$;

While dis satisfy the termination condition, do

Begin

$$
\begin{aligned}
& \text { Restructuring } p(t) \text {, obtain } c(t) \text {; } \\
& \text { Evaluate } c(t) \text {; } \\
& \text { Select } p(t+1) \text { from } p(t) \text { or } c(t) \text {; } \\
& t \leftarrow t+1 \text {; }
\end{aligned}
$$

end

end

\section{The concrete design of genetic algorithm}

\section{Gene expression.}

In this paper, a binary one-dimensional coding is used to determine the gene of $N$ nodes network, which is shown in Table. 1.

Table. 1 Network node gene

\begin{tabular}{|l|l|l|}
\hline $\mathrm{N}_{1}$ & $\mathrm{~N}_{2}$ & $\cdots$ \\
\hline $\mathrm{g}_{11}$ & $\mathrm{~g}_{22}$ & $\cdots$ \\
\hline$\cdots$ & $\cdots$ & $\cdots$ \\
\hline $\mathrm{g}_{1 \mathrm{n}}$ & $\mathrm{g}_{2 \mathrm{n}}$ & $\cdots$ \\
\hline
\end{tabular}

For example: the node gene expression of four-node network is shown in Table. 2, the network diagram of the node is shown in Fig. 1.

Table. 2 Network node gene of the example

\begin{tabular}{|c|c|c|c|}
\hline $\mathrm{N}_{1}$ & $\mathrm{~N}_{2}$ & $\mathrm{~N}_{3}$ & $\mathrm{~N}_{4}$ \\
\hline 0101 & 1010 & 0101 & 1010 \\
\hline
\end{tabular}




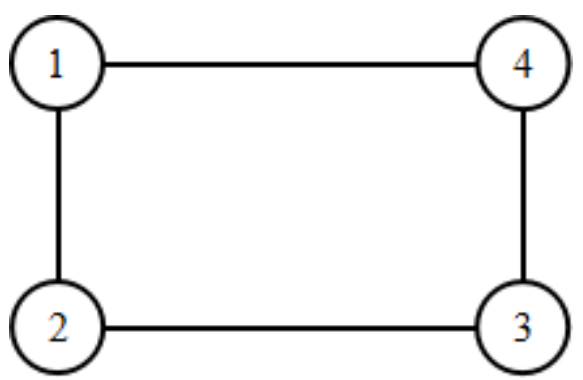

Fig. 1 Network diagram

\section{The selection of the fitness function.}

To effectively prevent GA deception occurring, the cost value of individuals in the population is arrayed in descending order.

the minimum cost of individual sorting encoding is set as 1 , and the cost of the largest individual sorting coding is set as $P_{0} P_{-}$size. Thus, the fitness function designed is as follows:

$$
f(x)=(x-1) /\left(P_{0} P_{-} \text {size }-1\right)
$$

Where $x$ represents the individual position in the sort of cost; $P o P_{-}$size represents the size of population, and $1 \leq x \leq P o P_{\text {_ size }}$.

\section{The selection operation.}

Use roulette selection, and the basic idea is that each gene selection probability is proportional to its fitness value. For the gene whose fitness value is $f_{k}$, its selection probability is calculated as follows:

$$
P_{k}=f_{k} / \sum_{j=1}^{\text {PoP_size }} f_{i}
$$

\section{Genetic operation.}

(1) Crossover

For network nodes, the randomized crossover node method is applied. Generate a random number in $[1, N]$, and the location of genes crossover is determined. Each time a node position can only be crossed. The crossover rate $P_{c}$ is normally set in $[0.01,0.1]$.

For example:

$\begin{array}{ccccccc}\text { Parent1 } & N_{1} & N_{2} & N_{3} & N_{4} & N_{5} & N_{6} \\ & 010110 & 101100 & 010101 & 101011 & 100101 & 001110 \\ \text { Parent2 } & N_{1} & N_{2} & N_{3} & N_{4} & N_{5} & N_{6} \\ & 010010 & 101100 & 011101 & 100111 & 100101 & 101110\end{array}$

The crossover operation is occurred in the third position, the progeny generated is shown as follows: 


$\begin{array}{ccccccc}\text { Progeny1 } & N_{1} & N_{2} & N_{3} & N_{4} & N_{5} & N_{6} \\ & 010110 & 101100 & 011101 & 101011 & 100101 & 001110 \\ \text { Progeny2 } & N_{1} & N_{2} & N_{3} & N_{4} & N_{5} & N_{6} \\ & 010010 & 101100 & 010101 & 100111 & 100101 & 101110\end{array}$

The crossover operation keeps the original connectivity of the network, but it is very likely to generate the gene which can not represent the correct structure of the communication network. Therefore, it is necessary to adjust the operating algorithm used to resolve such conflicts.

(2) Mutation

At first, it need to determine the genes to be mutated and theirs number. Then, choose a new gene to replace the original gene fragment within the specified range, as follows:

1) Set mutation gene as $x=\left[x_{1}, x_{2}, \cdots, x_{k}\right]$;

2) Randomly select integer $k \in[1, n], \quad \mu \in[1, n]$;

3) Generate progeny $x^{\prime}=\left[x_{1}, x_{2}, \cdots, x_{k}^{\prime}, \cdots, x_{k}\right]$, where $x_{k}^{\prime}$ is a uniformly distributed random value in $\left[x_{k}^{1}, x_{k}^{\mu}\right]$;

4) If the step three can not be achieved, jump to step two.

Under normal circumstances, the mutation rate $P_{m}$ is in $[0.001,0.01]$. If the mutation gene still produces incorrect configuration of network connectivity, it can be adjusted by adjusting the algorithm.

\section{Adjustment algorithm steps.}

Follow these steps to achieve the adjustment algorithm:

(1) Judge the network communication architecture of all the gene expression;

(2) If $g_{i j}=1$, implement the original crossover and mutation operation;

(3) If $g_{i j}=0$, set $g_{i j}=1$;

(4) If $g_{i j}=1$, set $g_{i j}=0$;

(5) If the step four can not be achieved, then jump to step one.

\section{Simulation example}

The following is a computer network reliability optimization calculation example. The mathematical model mentioned above, the cost matrix $C_{0}$ and reliability matrix $R_{0}$ of network link medium is expressed as Eq. (8) and Eq. (9). 


$$
\begin{aligned}
C_{0} & =\left[\begin{array}{cccccc}
0 & 5 & 8 & 12 & 14 & 7 \\
5 & 0 & 9 & 4 & 8 & 11 \\
8 & 9 & 0 & 5 & 10 & 9 \\
12 & 4 & 5 & 0 & 3 & 7 \\
14 & 8 & 10 & 3 & 0 & 6 \\
7 & 11 & 9 & 7 & 6 & 0
\end{array}\right] \\
R_{0} & =\left[\begin{array}{cccccc}
0 & 0.98 & 0.95 & 0.99 & 0.999 & 0.96 \\
0.98 & 0 & 0.97 & 0.988 & 0.996 & 0.99 \\
0.95 & 0.97 & 0 & 0.96 & 0.95 & 0.998 \\
0.99 & 0.988 & 0.96 & 0 & 0.93 & 0.92 \\
0.999 & 0.996 & 0.95 & 0.93 & 0 & 0.97 \\
0.96 & 0.99 & 0.998 & 0.92 & 0.97 & 0
\end{array}\right]
\end{aligned}
$$

The network nodes is set as 6 . The network node reliability constraint constant $\alpha$ and $\beta$ is set as 2 and 3. The iteration of genetic manipulation is set as 100 . After simulation calculation, the total cost of network link medium is 45 , and the network reliability has a maximum value 0.885 .

\section{Conclusions}

In this paper, genetic algorithm was applied to a computer network reliability optimization calculation, which reduced the network link media costs, improved network reliability. The proposed algorithm is simple and easy to implement program and readable. The simulation proved that genetic algorithms for solving these problems is of very practical value.

\section{References}

[1] Wu Xiaoyue, Zhang Weiming, Sha Jichang. With malfunction node's net reliability information's interact algorithms. National Defense Science and Technology University Journal, Volume 2 (1999), P. 76-80.

[2] Szczerbicka H, Beckcr M, Syrjakow M. Genetic algorithms:A tool for modeling,simulation and optimization of complex systems. Cybernetics and Systems, Volume 29, Issue 7 (1998), P. 639-659.

[3] Brown R E, Hanson A P, Willis H L, et al. Assessing the reliability of distribution systems. IEEE Computer Application Power, Volume 1, Issue 1 (2001), P. 44-49. 